\title{
ANÁLISIS Y REESTRUCTURACION DEL PROGRAMA DE UNA MATERIA UNIVERSITARIA, BAJO EL ENFOQUE DE FORMACIÓN DE COMPETENCIAS PROFESIONALES.
}

Ing. Romero, Lucrecia Natalia ${ }^{1}$

\section{RESUMEN:}

Este artículo propone la revisión de los programas vigentes de las materias universitarias, a la luz del enfoque de la formación basada en competencias profesionales.

Palabras claves: revisión, programa, fundamentación, objetivos, reestructuración, proyecto formativo.

En la edición anterior de la revista "EXTENSIONISMO, INNOVACIÓN Y TRANSFERENCIA TECNOLÓGICA-Claves Para E1 Desarrollo"-Volumen II Año 2015, se introdujeron las nociones básicas que persiguen este nuevo enfoque y propuestas, en la formación universitaria.

Los deseos de implementación, afloraron a partir de la realización del curso denominado "ENSEÑAR Y EVALUAR EN LA UNIVERSIDAD. REFLEXIONES $Y$ PROPUESTAS BASADAS EN EL ENFOQUE DE COMPETENCIAS" dictado por las profesoras SUSANA AVOLIO DE COLS Y SILVIA PALEY de la Universidad de Buenos Aires (UBA)

"La formación de una competencia permite a las personas que movilicen, apliquen e integren los conocimientos que han adquirido; en situaciones diversas, complejas eimpredecibles, se puede definir entonces la competencia como un saber hacer reflexivo y fundamentado" (1)

Es de sumo interés definir entonces las características del PROYECTO FORMATIVO en cuanto adquiere suma importancia su cohesión interna. Hablaremos de esta manera, de un PROYECTO FORMATIVO INTEGRADO(2), este concepto se aplica a distintos niveles en que se desarro1la la acción formativa, desde la elaboración de los planes de estudio hasta la programación de la enseñanza que realiza cada docente. Esto se refleja en los contenidos y actividades que cobran sentido en función de su coherencia con el proyecto total. 
En este enfoque se exalta la práctica profesional ya que permite identificar cuales son los saberes que se deben aprender, comprometiendo al docente en su elección y a los alumnos a aprender lo que no saben hacer, haciéndolo.

En primera instancia, se deberá analizar si el programa, se inscribe en el marco del perfil profesional y el perfil de egreso.

La elaboración del programa es el producto de la reflexión del equipo docente o de un docente en el que se expresan los principios y criterios que sostiene la Cátedra sobre la mejor forma de enseñar, la finalidad de la enseñanza, los contenidos, las pautas de organización de los trabajos, la metodología, y los criterios para la evaluación. ${ }^{(2)}$

En este caso particular de la FBC (FORMACIÓN BASADA EN COMPETENCIAS PROFESIONALES) se definirán los objetivos generales y específicos de la materia en términos de capacidades inferidas de las competencias. Se elegirán los contenidos en función de esas capacidades y se organizarán en torno a problemas, situaciones o tareas de la práctica en módulos/unidades/ bloques de contenidos. Asimismo, se especificarán los objetivos, los contenidos, propuesta de enseñanza, criterios de evaluación y carga horaria. Todo apuntará a desarrollar finalmente los requisitos de aprendizaje.

Como un primer acercamiento a este nuevo diseño curricular, se nos propuso diseñar un Módulo de la cátedra en la que cada docente es partícipe, para formularlo según la FBC.

De acuerdo a las consignas de las profesoras $^{(3)}$ :
Los aspectos a considerar en el diseño del módulo son los siguientes:

1. Título/Eje.

2. Introducción/fundamentación

3. Objetivos.

4. Contenidos.

5. Propuesta de enseñanza (trabajo integrador, estrategias de enseñanza,

secuencia didáctica ( técnicas, actividades formativas, medios)

6. Criterios para la evaluación

7. Carga horaria.

8. Requisitos de aprendizaje.

\section{Introducción/fundamentación}

En la introducción se describen los aspectos generales del módulo/unidad/bloque de contenidos y se fundamenta la propuesta.

Tiene que:

- Presentar la necesidad de inclusión del módulo/unidad/bloque de contenidos.

- Ubicarlo en la totalidad del Diseño Curricular.

- Expresar el propósito del mismo, su sentido para la formación profesional y la manera en que se vincula con las competencias del rol. 
- Indicar las relaciones que se pueden establecer con los otros módulos/unidades/bloques de contenidos

\section{Objetivos}

Expresan las capacidades requeridas para la resolución de situaciones problemáticas, consideradas críticas en términos formativos, en relación con el módulo/unidad/bloque de contenidos que se planifique. Se refieren a una actuación potencial, a la posibilidad de actuar en diversas situaciones, no limitándose a conductas específicas en contextos particulares.

\section{Contenidos}

Se refieren al qué enseñar y se definen como el conjunto de saberes o formas culturales cuya asimilación y apropiación por parte de los participantes contribuye al desarrollo de las capacidades requeridas.

El término hace referencia " $a$ conceptos, a procedimientos, a criterios, a normas y a valores que posibilitarán el desarrollo de capacidades relativas al conocer, el saber hacer y el saber ser que subyacen al desempeño competente en un determinado contexto."

\section{Actividades.}

Las actividades, tanto individuales como grupales, permiten concretar las estrategias y técnicas que se utilizan para orientar los procesos de aprendizaje y contribuir al logro de las capacidades requeridas. Pueden ser descriptas en términos del tipo de capacidad que se desarrolla, el número de participantes, el espacio físico en el que se realiza, los recursos y materiales empleados y la delimitación de segmentos temporales (iniciación, desarrollo y cierre) en el desarrollo del mó- dulo, de la unidad didáctica o de la clase.

\section{Criterios de evaluación}

Los criterios que se considerarán para evaluar las capacidades propuestas constituyen una referencia a partir de la cual se puede determinar si un alumno alcanzó o no los objetivos y cuáles son los requisitos mínimos para decidir la aprobación.

\section{Carga horaria}

La asignación de horas está relacionada con la complejidad del Módulo/unidad/bloque de contenidos.

Se pueden producir variaciones derivadas de las características de quienes participan, la necesidad de intensificar determinados aprendizajes y los recursos disponibles, entre otros aspectos.

Se sugiere:

a) Prever alrededor de un $10 \%$ del tiempo total del módulo/unidad/bloque de contenidos para actividades de apertura referidas a todo el módulo/uni$\mathrm{dad} /$ bloque de contenidos (planteo de problemas, casos o incidentes, presentación de un video o un marco conceptual a abordar, entre otras alternativas.).

b) Prever actividades de desarrollo para alrededor de 80\% del tiempo asignado al módulo/unidad/bloque de contenidos. Si se determinaron unidades y bloques de objetivos y contenidos dentro del módulo/unidad/ bloque de contenidos, distribuir ese $80 \%$ entre los mismos, integrando teoría y práctica.

c) Prever alrededor de un $10 \%$ del tiempo total del módulo/unidad/bloque de conteni- 
dos para actividades de cierre y evaluación

\section{Requisitos de aprendizaje}

El cursado de cada módulo/unidad/ bloque de contenidos requiere que quienes participan dispongan de saberes y experiencias que garanticen el nuevo aprendizaje, independientemente que éstos hayan sido adquiridos a través de capacitaciones formales o de la práctica profesional. En este sentido, será necesario precisar en forma muy concreta cuáles son los aprendizajes previos requeridos para cursar satisfactoriamente el Módulo/unidad/bloque de contenidos en cuestión.

Aquí definiremos, en referencia a la propuesta de actividades el concepto de secuencia didáctica, que apunta a un recorrido de enseñanza con coherencia, de tal manera que respondan a un fin claro. La secuencia didáctica se refiere a la organización en el tiempo de las técnicas de enseñanza, actividades de los alumnos y docente/s, contenidos trabajados para alcanzar objetivos propuestos. Comprende en forma interrelacionada la secuencia de enseñanza (técnicas y actividades que realiza el docente), y la de aprendizaje (actividades de aprendizaje que realizan los alumnos). Si bien se diferencian teóricamente, en la práctica, constituyen un proceso en el que docente y alumnos se comprometen para alcanzar determinados objetivos.

La estrategia de enseñanza y el trabajo integrador final cumplen la función de ser el hilo conductor de las actividades de la unidad o módulo.

El punto de partida para decidir cuá- les son las mejores técnicas y actividades son los objetivos establecidos en el módulo o unidad, el perfil del grupo y las características de los estudiantes, el/los contenido/s a enseñar y del contexto. ${ }^{(3)}$

A continuación se transmite el trabajo que realice en la materia en la que soy partícipe y que todavía sigue en análisis y transformación, según el avance del curso FBC.

\section{MATERIA: CARRETERAS (Carrera} Ingeniería Civil)

1. FUNDAMENTACIÓN DE LA MATERIA CARRETERAS:

Generar en los alumnos que no pertenecen a la orientación VIAS DE COMUNICACIÓN, las competencias apropiadas para el diseño, cálculo y control de la construcción, de una red vial, desde un análisis técnico-económico y social de su finalidad, como el de sus obras complementarias. Y como un caso particular aplicar dichas competencias en el diseño geométrico de un aeródromo. De esta manera, contribuye a desarrollar una de las incumbencias de la carrera: Estudio, factibilidad, proyecto, dirección, inspección, construcción, operación y mantenimiento de Obras viales Obras de urbanismo en lo que se refiere al trazado urbano y organización de servicios públicos vinculados con la higiene, vialidad, comunicaciones y energía. Planeamiento de sistemas de transporte en general y de tránsito en rutas y ciudades.

Acompañar a los alumnos en la búsqueda del perfil de profesional que demanda la sociedad, en relación al desarrollo socio-económico de un país, de manera de preservar y conservar los recursos naturales. Para ello; se pretende lograr que los alumnos construyan criterios de elección 
entre las distintas alternativas que surjan de la problemática planteada y en base al análisis reflexivo logren fundamentar esa elección a través de herramientas técnicosocio-económicas apropiadas. Se vincula con las materias TOPOGRAFÍA Y GEODESIA, que se dicta en el 2do Cuatrimestre de 2do año, TECNOLOGÁ DE LOS MATERIALES, 1er Cuatrimestre de 3er año, MECÁNICA DE SUELOS Y FUNDACIONES ,1er cuatrimestre de 4to año y FERROCARRILES A Y ECONOMIA, EQUIPOS Y ORGANIZACIÓN DE OBRAS, que se dictan en el 2do Cuatrimestre de 4to año de la carrera.

\section{OBJETIVO GENERAL :}

El alumno será capaz de planificar, diseñar y controlar la construcción de un camino, identificando los elementos a tener en cuenta, cuantificándolos, y calificándolos críticamente para obtener la solución más eficaz desde el punto de vista técnico-económico y social.

\section{OBJETIVOS ESPECIFICOS:}

3.1) Relevar, cuantificar y calificar críticamente todos los datos que puedan obtenerse para valorar la necesidad de un trazado vial que responda a las necesidades del contexto y consecuentemente, elaborar la estrategia más eficaz desde el punto de vista técnico-económico y social.

3.2) Diseñar geométrica y estructuralmente una red vial, teniendo en cuenta las medidas de seguridad requeridas para evitar futuros accidentes.

3.3) Diseñar, calcular y saber ubicar las obras complementarias de saneamiento, asumiendo la im- portancia de las mismas para el mantenimiento y conservación de las obras viales.

3.4) Evaluar críticamente el trazado elegidoy sus variantes; obteniendo los costos asociados y realizando una evaluación económica de los mismos fundamentada.

3.5) Seleccionar los materiales en función a su aptitud, sus características y magnitud de la obra, conociendo los avances tecnológicos y la aparición e innovación permanente de nuevos materiales y técnicas en el mercado. Realizar los ensayos de laboratorio de control de obra e interpretar los ensayos que no realice.

3.6) Desarrollar la capacidad de comunicación, trabajo en equipo, intercambio de opiniones y consenso. Elaborar un informe técnico que detalle las decisiones profesionalesadoptadasfundamentadas.

A continuación se presentan los distintos módulos a desarrollar:

Módulo 1: PLANIFICACION DE UNA RED VIAL QUE RESPONDA A LAS NECESIDADES DEL CONTEXTO.

Módulo 2: DISEÑO GEOMÉTRICO DE UN CAMINO.

Módulo 3: DISEÑO ESTRUCTURAL DE UN CAMINO.

Y como ejemplo, a continuación, se profundiza en el análisis del Módulo 1:

1. Título del módulo: Módulo 1: PLANIFICACION DE UNA RED VIAL QUE RESPONDA A LAS NECESIDADES DEL CONTEXTO 
2. Introducción del módulo: Se pretende en este módulo que el alumno desarrolle la capacidad de identificar los elementos básicos que componen una red vial y los factores a tener en cuenta en su planificación y diseño. La finalidad del módulo es que el alumno sea capaz de analizar críticamente la problemática profesional de la prefactibilidad de una obra vial, condición fundamental para el análisis de la factibilidad y diseño definitivos a adoptar, a través del desarrollo de los demás módulos.

\section{Objetivos}

OBJETIVO GENERAL: al finalizar el módulo el alumno será capaz de planificar un sistema de transporte, identificando los elementos a tener en cuenta, cuantificarlos, y calificarlos críticamente y de manera inicial obtener la solución más eficaz desde el punto de vista técnicoeconómico y socio-medioambiental. Con el desarrollo de los próximos módulos y al finalizar el curso, será capaz de revisar críticamente este análisis inicial adoptado.

OBJETIVOS ESPECIFICOS: al finalizar el módulo el alumno será capaz de:

> Identificar la necesidad de la construcción de una red vial determinada.

$>$ Cuantificar y calificar los datos relevados necesarios para la planificación de la red vial en referencia a los datos climatológicos, geográficos, topográficos, medioambientales.

$>$ Cuantificar el tránsito de la red vial y su capacidad.

$>$ Analizar e identificar los costos asociados a la construcción de la red vial.
$>$ Comparar varios trazados desde el punto de vista económico mediante una evaluación económica

$>$ Proponer un trazado fundamentando las decisiones adoptadas.

\section{Contenidos del módulo.}

A) UNIDAD $N^{\circ} 1$ : ¿Para qué fin se construye una red vial? ¿Qué elementos hay que tener en cuenta su planificación?

Análisis del contexto socio-económico-cultural. Conceptos generales. Elementos de un camino. Etapas en el proyecto de una red vial: Prefactibilidad, Factibilidad y Diseño Definitivo. Análisis en planta, perfil longitudinal y transversal.

Concepto de Tránsito. Como determinar el TMDA de un camino a través de distintos tipos de contadores de tránsito.

Concepto de Capacidad de un camino. Clasificación de volúmenes. Determinación de los niveles de servicio y posterior capacidad de un tramo de camino.

B) UNIDAD N²: ¿Cómo realizar un estudio de costos?

Identificar distintos tipos de costos. Usuarios, vehículos, comunidad según las normas COV de Vialidad Nacional. Corrección de costos según el caso particular a analizar.

C) UNIDAD N³: ¿Cómo realizar una comparación de trazados desde una evaluación económica de proyectos?

Lograr aplicar distintos índices y 
principios financieros para la elección eficaz de un trazado sobre otro.

\section{La propuesta de enseñanza:}

El trabajo integrador a plantear, abarcaría prácticamente todos los contenidos de la materia. Se propondría al inicio del dictado de la materia y se iría modificando, transformando y enriqueciendo; a lo largo del desarrollo de los tres módulos. La propuesta de la Cátedra CARRETERAS sería brindarles información a los alumnos sobre dos ciudades o puntos estratégicos donde se quiere construir un trazado vial que los vincule.

Para ello el alumno deberá identificar inicialmente la problemática dentro del contexto socio-económico-cultural para entender y poder analizar las distintas alternativas que podrían plantearse desde esas perspectivas. Esta sería una primera introducción para acercarlo al vocabulario vial y al problema a resolver utilizando los saberes previos y el sentido común. Una meta interesante a lograr es que estos alumnos que vienen de otras orientaciones, lograran encontrar en este primer estudio las coincidencias y diferencias que existen entre las obras viales y otras obras de índole civil o hidráulica con las que se encuentran más familiarizados. Asimismo, se perseguiría otra meta ambiciosa; que los alumnos mediante el análisis y la práctica reflexiva; se acercaran a los requerimientos del perfil profesional que exige la sociedad y su labor importante en el desarrollo de la misma.

De esta manera llegaríamos a identificar las cargas que afectan las obras viales "Tránsito" asociado a la "Capacidad" de un camino, elementos básicos como en toda obra, para su diseño.

Se propondrá a los alumnos que el tra- bajo sea grupal en comisiones de 2 personas y que se entregue por escrito un primer informe sobre las propuestas analizadas y los criterios para su elección.

Para el desarrollo de la unidad No2 referida al cálculo de los costos asociados a una red vial para analizar su viabilidad, se realizaría un sondeo a través de un cuestionario que apuntaría a conocer que conceptos han visto en la materia correlativa ECONOMIA, EQUIPOS Y ORGANIZACIÓN DE OBRAS, que se dictan en el 2do Cuatrimestre de 4to año de la carrera. Se les presentaría el Manual de COV (costo de operación de vehículos) de Vialidad Nacional y como utilizarlo. Posteriormente, los docentes nos encargaríamos de aclarar que el análisis de costos se desarrolla para determinadas características básicas del diseño del trazado y que en condiciones particulares se deberán hacer las correcciones correspondientes.

A modo de ejemplo se presentará para el desarrollo de la Unidad No 3 la evaluación económica de 2 trazados de una obra determinada y se propondrá que se realice dicha tarea entre 2 trazados que hayan propuesto inicialmente en las comisiones de 2 alumnos, presentándolo por escrito.

Como actividad final del módulo se propondrá que los distintos grupos expongan oralmente su trabajo, generando el intercambio de opiniones.

\section{Carga horaria del módulo:}

E1 módulo tendrá una duración de 18 horas (6 clases). Se planifica asignar 3 clases a la Unidad No 1,1 clase a la Unidad No 2, 1 clase a la unidad No 3 y 1 clase para la exposición oral de los grupos y el intercambio de opiniones, como cierre del primer módulo. 
La propuesta anteriormente expuesta; es un primer acercamiento de planificación según la FBC, como resultado de la realización de los tres primeros módulos del curso docente. Como consecuencia, se encuentra en constante revisión y a la espera del cursado del módulo 4, que seguirá enriqueciendo esta propuesta de enseñanza y se centrará

REFERENCIAS BIBLIOGRÁFICAS:

(1). APUNTES DEL CURSO: "ENSEÑAR Y EVALUAR EN LA UNIVERSIDAD. REFLEXIONES Y PROPUESTAS BASADAS EN EL ENFOQUE DE COMPETENCIAS” MODULO 1: Enseñar y evaluar en la universidad. Demandas y enfoques. La cátedra universitaria. Unidad 3. Dictado por las profesoras SUSANA AVOLIO DE COLS Y SILVIA PALEY de la Universidad de Buenos Aires (UBA)

(2). APUNTES DEL CURSO:"ENSEÑAR Y EVALUAR EN LA UNIVERSIDAD. REFLEXIONES Y PROPUESTAS BASADAS EN EL ENFOQUE DE COMPETENCIAS” MODULO 2: Cómo elaborar el programa de la cátedra .Unidad 1. Dictado por las profesoras SUSANA AVOLIO DE COLS Y SILVIA PALEY de la Universidad de Buenos Aires (UBA)

(3). APUNTES DEL CURSO:"ENSEÑAR Y EVALUAR EN LA UNIVERSIDAD. REFLEXIONES Y PROPUESTAS BASADAS EN EL ENFOQUE DE COM- específicamente en los criterios y formas de evaluación.

De esta manera y a modo de comprobación, el docente podrá evaluar si ha logrado implementar una secuencia didáctica coherente, que se traduzca en el saber hacer fundamentado de sus alumnos.

PETENCIAS” MODULO 3: Estrategias de enseñanza y entornos educativos. Unidad 4. Dictado por las profesoras SUSANA AVOLIO DE COLS Y SILVIA PALEY de la Universidad de Buenos Aires (UBA)

(4) "Construir competencias desde la escuela”. PERRENOUD PHILLIPE (2003),J.C.Saez, editor, Chile.

(5) "Las competencias docentes del profesorado universitario: calidad y desarrollo profesional". ZABALZA BERAZA, M.A (2003). Madrid. Narcea.

(6) "La cabeza bien puesta. Bases para una reforma educativa". MORIN, E. (2002), Buenos Aires, Nueva Visión.

(7) "Prácticas Innovadoras en la formación de docentes universitarios”. LUCARELLI, ELISA.

Educação Porto Alegre - RS, ano XXVII, n. 3 (54), p. 503 - 524, Set./Dez. 2004

(8) "La enseñanza y los profesores: metáforas, modelos $\mathrm{y}$ formas de enseñar”. COLS, E: en Revista del IICE Año IX, No 17, diciembre de 2000 